\title{
Micromanagement of the ovarian follicle reserve - do stem cells play into the ledger?
}

Like bell-bottoms and The Beatles in the 1960s, the rage of the day here in the new millennium has become stem cells. Unlike the Cultural Revolution 40 years ago, stem cell mania is captivating the attention of the general populace as the biomedical research community announces breakthrough after breakthrough with promises of disease cures and now the power to manipulate the building blocks of procreation, namely gametes. Fresh on the heels of studies showing that embryonic stem cells can be coaxed into making either oocytes (Hübner et al. 2003) or sperm (Geijsen et al. 2004) comes the paper in the 11 March 2004 issue of Nature by Johnson et al. implying the existence of germline stem cells in the mouse ovary which may serve to replenish the supply of ovarian follicles postnatally. Revisiting old problems and challenging doctrine with new approaches is the esprit de corps of any scientific endeavor and the Tilly group is to be commended for their work and the public perception it has shaped regarding the mechanisms underlying ovarian senescence. Speaking of senescence, history does repeat itself and needs to be examined if this work turns out to overturn dogma as has been suggested. Simply stated, the biological problem has always been one of the economy of the ovarian follicle reserve - and there is little dispute over the fact that follicle attrition is a hallmark of the aging mammalian ovary. The question raised by Johnson et al. is whether a stem cell exists in the surface epithelium of the mouse ovary that gives birth postnatally to new follicles.

We might all benefit every once in a while from a visit to the 'old' literature and I especially suggest dusting off The development of the mouse ovary from birth to maturity (Peters 1969), in which Helen Peters cogently updates the historical argument around why the ovarian epithelium is indeed not at all 'germinal'or, by today's standards, 'stemmish'. She proceeds to show that patterns of tritiated thymidine incorporation are inconsistent with a postnatal mitotic origin of germ cells but clearly consistent with a stem-like character in the ovarian somatic cells that contribute to various cell lineages in the mature ovary. Experimental evidence supportive of an ovarian stromal stem cell source for somatic cell types in rat and mouse ovary arrived in 2000 (Eppig \& Wigglesworth 2000).

How should we proceed and can guidance be obtained from the experimental approaches embodied in the Johnson et al. study? What follows below is an attempt to sketch a possible road map for soliciting input and encouraging discourse for some of the points raised in this work that warrant close scrutiny. Although by no means complete, it is hoped that a purpose and direction will derive from these very basic inquiries given that the authors have diligently and cogently delivered their conclusion that an ovarian stem cell with germ line potential exists in the mouse ovary.

\section{Microeconomics of the ovarian follicle reserve}

The prompt elicited by the authors because the expected rate of follicle loss in three mouse strains did not match what they observed raises immediate concerns regarding the validity of assays for follicle atresia. The histomorphometric assay in the supplemental methods pinpoints oocyte degeneration as the hallmark that a follicle is atretic. But if follicles are 'atretic' before overt oocyte degeneration, estimates of the healthy follicle fraction are likely to be over-represented. Stereological methods applied to fixed tissues unfortunately fail to reveal tissue dynamics and the possibility that the commitment to atresia is not linear begs the question; How do 'static' snapshots relate to the actual kinetics of follicular recruitment, survival and demise?

\section{Follicle replenishment by newborn oocytes implies a dynamic process}

The germ cell marker vasa was shown to be expressed in ovarian epithelial cells that incorporate BrdU; that vasapositive epithelial cells are also shown to undergo mitosis (Johnson et al. 2004, Figure 2) is proffered to support the idea that oocyte and follicle renewal occur postnatally. How certain are we that oogonial proliferation and meiotic entry do not occur postnatally? This is well known to occur in hamsters, rabbits and other mammals. What age animals were used to generate this data? The authors report "juvenile and young adult mouse ovaries"; are meiotic entry markers (SCP3 positive cells; Johnson et al., Figure 3) more likely to be found in greater abundance in 4-day- versus 40-day old animals, when RNA sampling of whole tissue shows waning levels of other meiotic entry markers? (Supplemental methods). Has the vectorial pattern of germ cells in the epithelium been established i.e. are these on the way into the ovary or being released from the ovary? Like the economy of membrane turnover in cells, where exocytosis and endocytosis are balanced processes, sorting out the economy of follicles will require the use of new experimental approaches. While Johnson et al. favor the idea of de novo formation of germ cells in 
the ovarian epithelium, it seems possible that a fetal stem cell could home in from either vascular or peritoneal sites or even that their images capture the release of oocytes from the ovary. Indeed, the latter was clearly evidenced in the work of Peters (1969) but somehow has escaped the attention of some.

\section{Microchimerism after transplantation}

Green oocytes within follicles that are granulosa negative but thecal stromal positive (Johnson et al. 2004, Figure 5) provide evidence consistent with the idea that, after transplantation, follicles are formed from donor (GFP + ) germ line, host granulosa (GFP - ), and donor stroma (GFP + ). While conceptually simple, these results are difficult to reconcile and alternative explanations may exist. Are the follicles truly chimeric and why is there a separation of germ line, stroma and granulosa lineages? The concept of ovarian somatic stem cell was proposed 40 years ago by Peters (1969) and, as noted above, recent studies reveal the plasticity of germ and somatic lineages in rat/mouse chimeric ovaries (Eppig \& Wigglesworth 2000). Is migration of primordial follicles from GFP tissue into host tissue a more likely explanation for this result? Are differences in allelic expression of the GFP transgene and/or differences in the nucleocytoplasmic volume of oocytes, granulosa and theca likely to influence the interpretation of these results? Time will tell.

The community of reproductive biologists must now responsibly marshal a systematic effort aimed at confirming, extending and/or challenging the data upon which these conclusions have been based. The healthy sense of skepticism permeating laboratories vested in the mysteries of ovarian biology should encourage new lines of investigation and a vigorous outpouring of results submitted for peer review in specialty journals like Reproduction. In the meantime, it is imperative that data bearing directly on the existence of ovarian stem cells be collected and that the potential significance of this work not be taken lightly by reproductive biologists in general and those fascinated by the biology of stem cells. Whether new germ line derivatives become incorporated into a postnatally refreshed follicle reserve is a most welcomed query as this Mother Nature riddle begs for rhyme and reason.

D FAlbertini (david.albertini@tufts.edu) Department of Anatomy \& Cellular Biology

Tufts University School of Medicine

136 Harrison Avenue

Boston MA 02111

USA

\section{References}

Hübner K, Fuhrmann G, Christenson LK, Kehler J, Reinbold R, De La Fuente R et al. 2003 Derivation of oocytes from mouse embryonic stem cells. Science $\mathbf{3 0 0} 1251-1256$.

Geijsen N, Horoschak M, Kim K, Gribnau J, Eggan K \& Daley GQ 2004 Derivation of embryonic germ cells and male gametes from embryonic stem cells. Nature 427 148-154.

Johnson J, Canning J, Kaneko T, Pru JK \& Tilly JL 2004 Germline stem cells and follicular renewal in the postnatal mammalian ovary. Nature 428 145-150.

Peters H 1969 The development of the mouse ovary from birth to maturity. Acta Endocrinologica 62 98-116.

Eppig JJ \& Wigglesworth K 2000 Development of mouse and rat oocytes in chimeric reaggregated ovaries after interspecific exchange of somatic and germ cell components. Biology of Reproduction 63 1014-1023. 